\title{
High-pressure molecular phases of solid carbon dioxide
}

\author{
S. A. Bonev, F. Gygi, T. Ogitsu, and G. Galli \\ Lawrence Livermore National Laboratory, University of California, Livermore, California 9455 * $^{*}$
}

(Dated: September 21, 2018)

\begin{abstract}
We present a theoretical study of solid carbon dioxide $\left(\mathrm{CO}_{2}\right)$ up to $50 \mathrm{GPa}$ and $1500 \mathrm{~K}$ using firstprinciples calculations. In this pressure-temperature range, interpretations of recent experiments have suggested the existence of $\mathrm{CO}_{2}$ phases which are intermediate between molecular and covalentbonded solids. We reexamine the concept of intermediate phases in the $\mathrm{CO}_{2}$ phase diagram and propose instead molecular structures, which provide an excellent agreement with measurements.
\end{abstract}

PACS numbers: 62.50.+p, 64.70.Kb, 61.50.Ah, 78.30.-j

Understanding the evolution of the bonding properties of molecular crystals as a function of pressure is a fundamental question in condensed matter physics. Recently, this question has received a widespread attention due to key progress in experimental techniques and hence, the availability of new data for a number of molecular solids 1]. In the case of $\mathrm{CO}_{2}$, the interest has been further intensified due to its importance for planetary science and technical applications. However, despite several experimental investigations of the $\mathrm{CO}_{2}$ high-pressure phases, the changes of intra- and inter-molecular bonds, as well as of electronic and vibrational properties, as a function of pressure are not yet well understood. In addition, large portions of the $\mathrm{CO}_{2}$ phase diagram are unexplored from a theoretical standpoint.

At low pressure $(P)$ and temperature $(T), \mathrm{CO}_{2}$ condenses as a molecular solid in the cubic Pa3 structure (known as dry ice, phase I), which is characterized by strong double bonds $(\mathrm{C}=\mathrm{O}$ distance of $1.16 \AA)$ and rather weak inter-molecular interactions 2]. Recently, a high- $P$ and high- $T$ phase of $\mathrm{CO}_{2}$ (phase $\mathrm{V}$ ) has been discovered [3, 4, 5, 6, 7], which is completely different from the molecular solid. It has a polymeric quartz-like structure, and a very low compressibility (the experimentally derived bulk modulus, $B_{0}$, is $365 \mathrm{GPa}$ ). Following this exciting discovery, two additional new phases have been reported [8, 9, 10] experimentally (II and IV in Fig. 11), both of which were credited with unusual properties; their structures were described as intermediate between that of molecular and covalently bonded crystals.

In this Letter, we present a study of the $\mathrm{CO}_{2}$ phase diagram based on first-principles density functional theory (DFT) calculations. Our results challenge the interpretation of $\mathrm{CO}_{2}$-II, III, and IV as exhibiting dramatic differences in the nature of the molecular bonding with respect to the low-pressure molecular crystal. In place of the previously proposed structures for $\mathrm{CO}_{2}$-II and IV, we suggest new, molecular ones. We demonstrate that in addition to being stable, our newly proposed structures give results in excellent agreement with measurements and provide a consistent explanation of experimental observations.

At ambient $T, \mathrm{CO}_{2}$-I undergoes a pressure-induced transformation to the orthorhombic $\mathrm{Cmca}$ symmetry (phase III) between 12 and $22 \mathrm{GPa} 3,11,12,13,14,15$, 16]. The exact nature of this transition and the structure of phase III are still unsettled; experimental studies have suggested a region of co-existence between $\mathrm{CO}_{2}$-I and III 14], and also an intermediate distorted low- $T$ phase [16]. Furthermore, it was reported [9] that the I to III transition is strongly temperature dependent, becoming abrupt above $400 \mathrm{~K}$, and that $\mathrm{CO}_{2}$-III may actually be metastable. The two newly discovered phases II and IV are believed to be thermodynamically stable and quenchable. It was proposed that $\mathrm{CO}_{2}$-II has the $P 4_{2} / m n m$ symmetry with elongated $\mathrm{C}=\mathrm{O}$ bonds, $1.33 \AA$, reduced inter-molecular distances below $2.38 \AA$ (hence named associated or dimeric phase), and $B_{0}=131 \mathrm{GPa}$ 9, 10]. $\mathrm{CO}_{2}$-IV was described as $\mathrm{Pbcn}$, with $\mathrm{C}=\mathrm{O}$ bonds as long as $1.53 \AA$, and bent $\left(\mathrm{O}=\mathrm{C}=\mathrm{O}\right.$ angle of $\left.160^{\circ}\right)$ and strongly (dipole) interacting molecules [8]. Such remarkable properties would imply that $\mathrm{CO}_{2}$ is losing its molecular character in a "gradual" way, a concept promoted as a key for understanding its phase diagram in analogy with solids like $\mathrm{N}_{2} \mathrm{O}$ and $\mathrm{SiO}_{2}$. In addition, $\mathrm{CO}_{2}$-III, despite having a stable $\mathrm{C}=\mathrm{O}$ bond, was also assigned a $B_{0}$ as high as $87 \mathrm{GPa}[9]$. The stability of the proposed bent and associated phases, and the unusual bulk properties of $\mathrm{CO}_{2}$-II, III, and IV have not been investigated theoretically up to date.

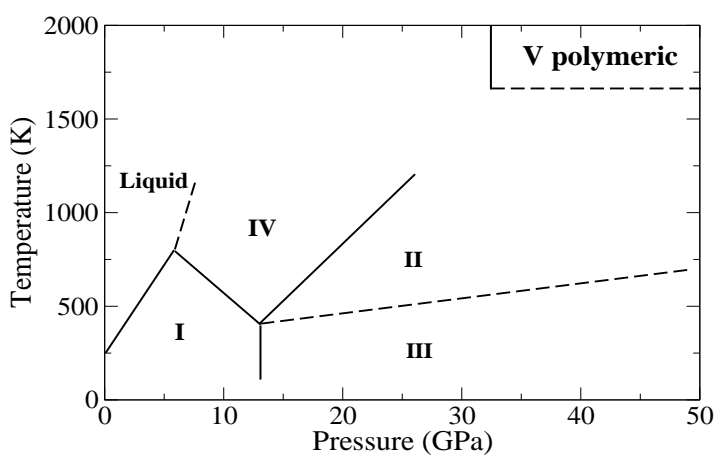

FIG. 1: Phase diagram of $\mathrm{CO}_{2}$ according to Ref 9, where $\mathrm{CO}_{2}$-III $(\mathrm{Cmca})$ is reported to be metastable. 
We performed a series of first-principles calculations, including full structural optimizations, phonon spectra, and free energies, in order to study the stability and properties of the phases proposed experimentally up to $50 \mathrm{GPa}$ and $1500 \mathrm{~K}$. The DFT calculations were carried out within the Perdew-Burke-Ernzerhof (PBE) 17] generalized gradient approximation (GGA) using the ABINIT code [18], which implements plane-wave basis sets 19.

First, we have examined the stability of the bent and dimeric structures for phases II and IV. Starting from the previously proposed $P 4_{2} / m n m$ and $P b c n$ with elongated molecular bonds, we carried full structural optimizations at various pressures up to $50 \mathrm{GPa}$. In all cases, upon relaxing the atomic coordinates the $\mathrm{C}=\mathrm{O}$ bond lengths decreased by about $15 \%$ and $30 \%$ for $P 4_{2} / m n m$ and $P b c n$ respectively, to become comparable to the free $\mathrm{CO}_{2}$ molecule [20]. In the case of $\mathrm{Pbcn}$, the $\mathrm{O}=\mathrm{C}=\mathrm{O}$ angle also straightened to $180^{\circ}$. The energy differences between the theoretically stable molecular structures and the associated and bent phases are respectively more than 3 and $6 \mathrm{eV}$ per molecule. These are energy scales corresponding to the breaking of a covalent bond. They are beyond the errors of the GGA and present a strong evidence that the previously proposed bent and dimeric structures are not stable. In order to further investigate this issue, we examine below published experimental data for $\mathrm{CO}_{2}$-II, III, and IV, and demonstrate that they can be explained in terms of two stable structures, $\mathrm{Cmca}$ and $\mathrm{P}_{2} / \mathrm{mnm}$, which are strictly molecular.

Computed equation of state (EOS) at $T=0$ for the three molecular structures $\mathrm{Pa} 3, \mathrm{Cmca}$, and $\mathrm{P}_{2} / \mathrm{mnm}$ are reported in Fig. 2 along with the available experimental data. In all three cases, the calculated EOS agrees very well with the measured one; the computed $P V$ curves fall within the experimental uncertainties. We find about $2 \%$ relative volume reduction from $\mathrm{Pa} 3$ to Cmca [21] above $30 \mathrm{GPa}$ and another $0.5 \%$ from $\mathrm{Cmca}$ to $P 4_{2} / \mathrm{mnm}$ (see insert in Fig. 2). Yoo et al. reported $\mathrm{a} \sim 5-7 \%$ volume decrease associated with the III to II transition; however, they also observed lattice strain in their phase III samples 9], which may explain the difference between their results and ours. Furthermore, the $P V$ curve of $\mathrm{Cmca}$ comes close to and eventually merges with that of Pa3 below $20 \mathrm{GPa}$ in agreement with Aoki et al. 14]. Thus, the measured EOS data are reproduced well despite the lack of molecular association in our optimized $P 4_{2} / m n m$. The computed EOS parameters are summarized in Table【together with values derived form experiment 22]. The agreement is good for Pa3, but there is an order of magnitude difference for the values of $B_{0}$ of $C m c a$ and $P 4_{2} / m n m$. Since we are using the same EOS fit as Ref. 10, and we in fact agree very well with the direct $P V$ measurements, the large difference likely comes from extrapolating the experimental data, spread above $20 \mathrm{GPa}$, down to ambient pressure.

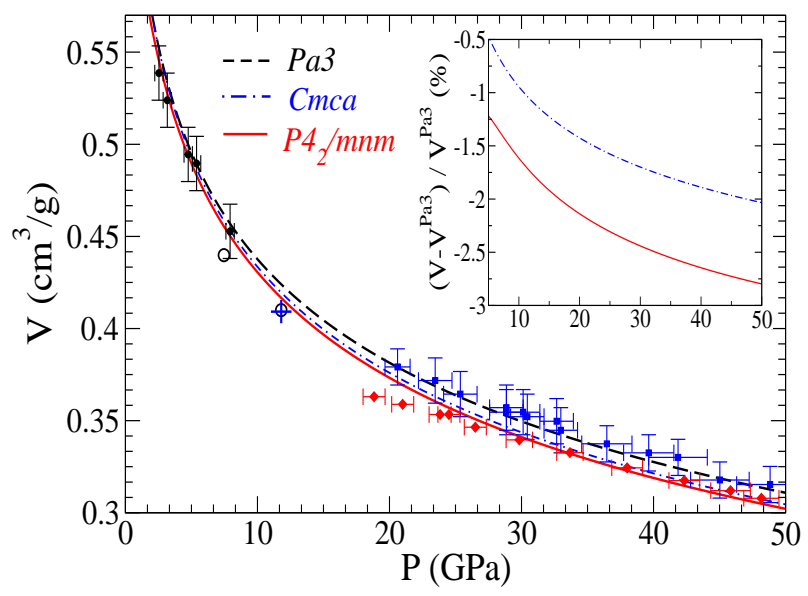

FIG. 2: Pressure-volume dependence of selected $\mathrm{CO}_{2}$ structures: Pa3 (black), Cmca (blue), and $P 4_{2} / m n m$ (red). The solid lines are $a b$ initio calculations; the points indicate experimental data from Ref. 14 (no error bars), Ref. 3 (Pa3 and $\mathrm{Cmca})$, and Ref. $10\left(P 4_{2} / \mathrm{mnm}\right)$. The inset shows calculated reduction in volume of $P 4_{2} / \mathrm{mnm}$ and $\mathrm{Cmca}$ relative to $\mathrm{Pa} 3$.

The published experimental evidence invoked to propose that $\mathrm{CO}_{2}$-II exhibits the properties of a dimeric polymorph consists of: ( $i)$ a large splitting of the internal symmetric stretching mode, $\nu_{1}[9],(i i)$ a broad librational mode identified as $\mathrm{B}_{1 g}$ [9, [10], and (iii) powder x-ray diffraction measurements 10]. We carried out calculations of the vibron spectrum [23] of molecular $\mathrm{P}_{2} / \mathrm{mnm}$ and $C m c a$ as a function of $P$. A plot of $\nu_{1}$ in Fig. 3 shows that the measured splitting, as well as the relative values for $P 4_{2} / m n m$ and $C m c a$, are reproduced remarkably well by the theoretical structures. Yoo et al. assumed that the large vibron splitting observed in $\mathrm{CO}_{2}$-II is an evidence for decreased inter-molecular distances; our results indicate that the crystal field in the molecular $\mathrm{P}_{2} / \mathrm{mnm}$ is sufficient to explain the splitting. One should note that phonons are calculated as a second derivative of the energy; therefore the agreement with experimental frequencies within $\mathrm{meV}$, as found here, is a strong indication that computed total energies and forces are extremely accurate.

The computed pressure dependence of the Raman-

TABLE I: Equation of state parameters for selected $\mathrm{CO}_{2}$ phases. The theoretical data is well fitted to a third-order Birch-Murnaghan equation of state.

\begin{tabular}{lcccc}
\hline \hline Structure & $V_{0}\left(\mathrm{~cm}^{3} / \mathrm{g}\right)$ & $B_{0}(\mathrm{GPa})$ & $B_{0}^{\prime}$ & Ref. \\
\hline$P a 3$ & 0.714 & 2.93 & 7.8 & 11 \\
$P a 3$ & 0.726 & 3.21 & 8.10 & this study \\
\hline Cmca & 0.450 & 87 & 3.3 & 10 \\
Cmca & 0.725 & 3.53 & 7.12 & this study \\
\hline$P 4_{2} /$ mnm & 0.408 & 131 & 2.1 & 10 \\
$P 4_{2} /$ mnm & 0.701 & 4.37 & 6.66 & this study \\
\hline \hline
\end{tabular}



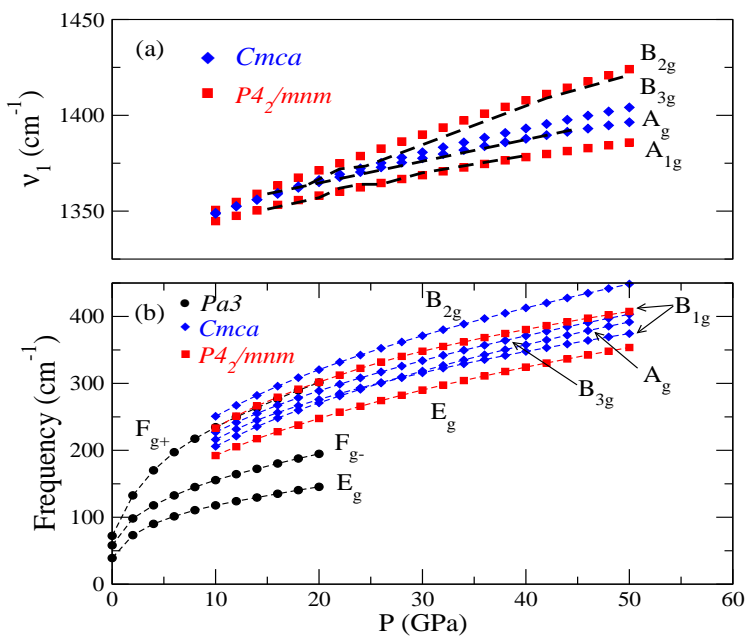

FIG. 3: Computed Raman-active modes (solid symbols) of the theoretically stable structures. (a) Symmetric stretching vibron. The dashed lines indicate experimental data from Ref. 9; they are from a Fermi resonance band, and have been shifted by $52 \mathrm{~cm}^{-1}$ for comparison. (b) External modes.

active external modes of the three theoretical structures is shown in Fig. 3b. The agreement with experiment 16 ] is again good, though our values are consistently slightly lower than the measured ones. The experimental frequencies of the two Raman-active modes of $\mathrm{CO}_{2}$-II at $19 \mathrm{GPa}$ are about 260 and $320 \mathrm{~cm}^{-1}$ - the former being a broad peak - and were classified as $\mathrm{B}_{1 g}$ and $\mathrm{E}_{g}$ respectively [9, 10]. The broad mode was previously associated with dynamical disorder in the lattice. The molecular $\mathrm{P}_{2} / \mathrm{mnm}$ has modes with frequencies 245 and $300 \mathrm{~cm}^{-1}$ at this $P$; however it is the degenerate $\mathrm{E}_{g}$ which has the lower frequency, and therefore corresponds to the experimentally observed broad peak. Its broadening can be explained by invoking a lifting of the $\mathrm{E}_{g}$ degeneracy when the tetragonal cell is deformed into an orthorhombic one (resulting in the Pnnm symmetry). Such a distortion is consistent with the x-ray data reported in Ref. 10 and we estimate that between 20 and $30 \mathrm{GPa}$ there is $10 \mathrm{~cm}^{-1}$ splitting for every $1 \%$ modifiaction of $a$ and $b$.

We now turn our attention to the x-ray analysis. In $\mathrm{P}_{2} / \mathrm{mnm}$, the atomic positions are $\mathrm{C}(2 \mathrm{a})$ at $[0,0,0]$, and $\mathrm{O}(4 \mathrm{f})$ at $[x, x, 0]$. The associated phase, according to Ref. 10 , corresponds to $x=0.2732$, while our stable structure corresponds to $x=0.23075$, i.e. $x \approx 0.25 \pm \delta$ for both structures, where $\delta=0.02$. Among the observed diffraction peaks, only the (101) and (211) reflections depend on the sign of $\delta$; their measured intensities relative to the calculated intensities of the same reflections are in ratios of 2.3 and 0.3 for the associated, and 0.4 and 1.8 for the molecular structures respectively [24]. We therefore conclude that at present the diffraction measurements are insufficient to distinguish between the proposed dimeric and the theoretical molecular $P 4_{2} / m n m$ structures.
Finally, we examine the relative stability of all considered crystal structures as a function of both $P$ and $T$. A plot of enthalpies showing that Cmca is never thermodynamically stable at low $T$ is reported in Fig. 4a. This finding confirms a previous observation by Iota and Yoo [4] that the orthorhombic symmetry obtained during ambient $T$ compression of $\mathrm{CO}_{2}$-I is metastable; the reason why the system reverts to it is the low kinetic barrier associated with the rotation of the molecules from their alignment in $\mathrm{Pa} 3$ to that in $\mathrm{Cmca}$. In additional support of this conclusion, we have performed structural optimizations starting from the $\mathrm{Pbca}$ structure, and relaxing the atomic coordinates. The Pbca lattice is obtained from $\mathrm{Pa} 3$ by deforming the cubic cell; further rotations of the molecules in the $y-z$ plane then leads to Cmca. In all such calculations above $15 \mathrm{GPa}$, the $\mathrm{CO}_{2}$ molecules quickly align in the $y$ - $z$ plane, thus ending up in the $C m c a$, rather than in the energetically favorable $P 4_{2} / \mathrm{mnm}$ phase. This happens regardless of whether the unit cell is tetragonal or orthorhombic; the deformation of $\mathrm{P}_{2} / \mathrm{mnm}$ to the orthorhombic Pnnm costs only about $1 \mathrm{meV}$ per molecule for $1 \%$ distortion of $a$ and $b$.

The kinetic barrier between $C m c a$ and $P 4_{2} / m n m$ can be overcome by heating the system above ambient $T$. Interestingly, we find that upon further heating at constant $P$ there is a thermodynamic phase boundary above which the orthorhombic phase is now stable. The phonon free energies, computed as described in Ref. 23, are plotted in Fig. 4b. The transition to Cmca is entropy driven and is
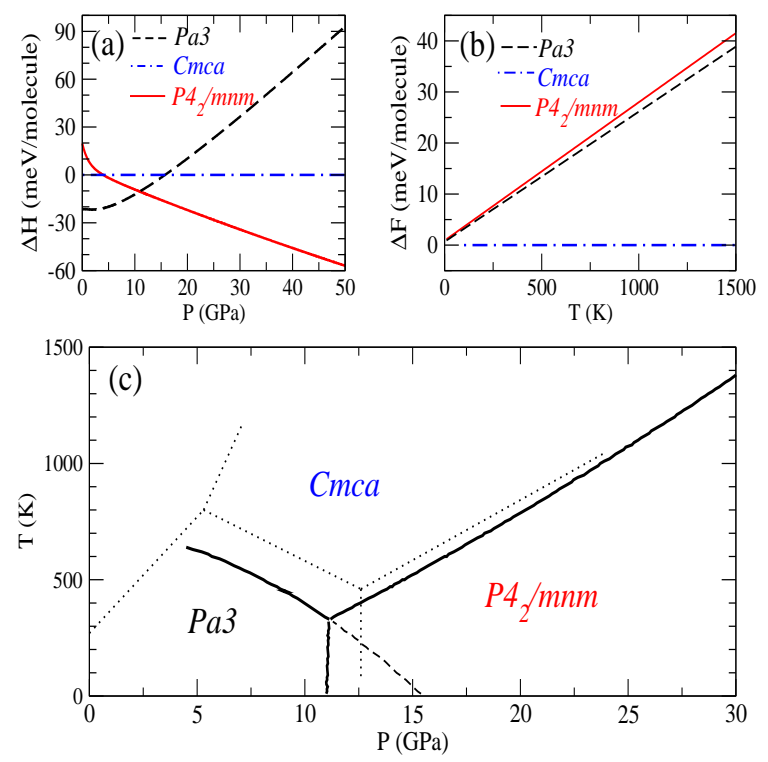

FIG. 4: (a) Enthalpy versus $P$ relative to the $C m c a$ phase at $T=0$. (b) Phonon free energy versus $T$ relative to the Cmca structure at $16 \mathrm{GPa}$; the reported differences do not vary strongly with $P$. (c) Relative stability of selected $\mathrm{CO}_{2}$ structures from Gibbs free energy comparison; here $P$ includes phonon contributions. The dotted lines indicate the experimental phase diagram constraints from Ref. 9 (see Fig. 1). 
mainly due to a soft acoustic phonon mode. Its physical origin is in the specific geometry of $\mathrm{Cmca}$, allowing for a relatively unobstructed shear-like motion parallel to the $y$ axis; we have confirmed this by sampling the potential surface with frozen-phonon calculations.

The computed stability regions for $\mathrm{Pa} 3, \mathrm{P} 4_{2} / \mathrm{mnm}$, and $\mathrm{Cmca}$ are shown in Fig. 4. Since we are comparing the relative stability of a limited number of structures, we cannot firmly conclude that Fig. 4 represents the actual phase diagram of $\mathrm{CO}_{2}$. However, the almost perfect matching of the stability region of $\mathrm{Cmca}$ with that of $\mathrm{CO}_{2}$-IV makes the identification between the two phases rather compelling. We note that a phase IV of $\mathrm{CO}_{2}$ was first suggested by Olijnyk and Jephcoat 16] based on the observed multiplicity of external Raman-active modes between 10 and $20 \mathrm{GPa}$, at ambient $T$. Their data is consistent with the Raman spectrum of Pa3, Cmca, and $P b c a$. As we discussed above, $P b c a$ is intermediate between $\mathrm{Pa} 3$ and $\mathrm{Cmca}$, and may be present close to the boundary of stability between these two phases, which extends from 5 to $16 \mathrm{GPa}$ in our calculations. In addition, $C m c a$ has a soft (below $100 \mathrm{~cm}^{-1}$ at $\sim 20 \mathrm{GPa}$ ) zone-boundary libron, $\mathrm{B}_{1 g}$, tilting the molecules away from the $y-z$ plane, which crosses two of the acoustic modes. Thus, when excited at high T, Cmca could actually turn into a peculiar distorted phase. Ref. 16 observed a similar distorted phase with a weak (otherwise forbidden) Raman activity of the bending internal mode, which they related to the presence of pressure inhomogeneities in the sample. We find such an explanation of the Raman activity observed by Yoo et al. [8] around $650 \mathrm{~cm}^{-1}$ more plausible than the existence of bent molecules.

In conclusion, we have presented a new interpretation of experimental data recently obtained for $\mathrm{CO}_{2}$-II, III, and IV. Our ab inito calculations identify $\mathrm{CO}_{2}$-II as a molecular structure with the $P 4_{2} / m n m$ symmetry. We have also elucidated the high- $T$ behavior of molecular $\mathrm{CO}_{2}$, and have found the $C m c a$ symmetry to be preferable due to its relatively large entropy. Based on our findings, we propose that measurements of the high- $T$ phase IV be interpreted along the lines of an orthorhombic, and possibly distorted, phase. Finally, we note that the external Raman modes of several $\mathrm{CO}_{2}$ molecular structures are very close in frequency (see Fig. 3), and therefore may not be sufficient to discriminate between different phases.

We thank C.S. Yoo, J. Park, and V. Iota for useful discussions. This work was performed under the auspices of the U.S. Dept. of Energy at the University of California/LLNL under contract no. W-7405-Eng-48.

* Electronic address: bonev1@llnl.gov

[1] See, for example, R.J. Hemley, Annu. Rev. Phys. Chem. 51, 763 (2000), and references therein.
[2] W.H. Keesom and J.W.L. Kohler, Physica 1, 167 (1934); P.W. Bridgeman, Proc. Amer. Acad. Arts Sci.; R.C. Hanson and L.H. Jones, J. Chem. Phys. 75, 1102 (1981); B. Olinger, J. Chem. Phys. 77, 6255 (1982).

[3] C.S. Yoo et al., Phys. Rev. Lett. 83, 5527 (1999).

[4] V. Iota, C.S. Yoo, and H. Cynn, Science 283, 1510 (1999).

[5] S. Serra et al., Science 284, 788 (1999).

[6] J. Dong et al., Phys Rev B 61, 5967 (2000); ibid. 61, 5967 (2000); Science 287, 11a (2000).

[7] B. Holm et al., Phys. Rev. Lett. 85, 1258 (2000).

[8] C.S. Yoo, V. Iota, and H. Cynn, Phys. Rev. Lett. 86, 444 (2001); BAPS 48, No. 1, 914 (2003), and Ref. 24.

[9] V. Iota and C.S. Yoo, Phys. Rev. Lett. 86, 5922 (2001).

[10] C.S. Yoo et al., Phys. Rev. B 65, 104103 (2002).

[11] L. Liu, Earth Planet Lett. 71, 104 (1984).

[12] R.C. Hanson, J. Phys. Chem. 89, 4499 (1985).

[13] B. Kuchta and R.D. Etters, Phys Rev B 38, 6265 (1988); 47, 14691 (1993).

[14] K. Aoki et al., Science 263, 356 (1994); Phys. Rev. B 48, 9231 (1993).

[15] R. Lu and A. M. Hofmeister, Phys. Rev. B 52, 3985 (1995).

[16] H. Olijnyk and A.P. Jephcoat, Phys. Rev. B 57, 879 (1998).

[17] J.P. Perdew, K. Burke, and M. Ernzerhof, Phys. Rev. Lett. 77, 3865 (1996).

[18] The ABINIT code is a common project of the Université Catholique de Louvain, Corning Incorporated, and other contributors (URL http://www.abinit.org).

[19] We used non-local pseudopotentials ( $\mathrm{s}$, p, and d angular momentum projections) of the Troullier-Martins type (N. Troullier and J.L. Martins, Phys. Rev. B 43, 1993 (1991)). A 45 Ha plane-wave cut-off and $4 \times 4 \times 4$ Monkhorst-Pack (MP) grids were found sufficient for energy, pressure, and bond length converged better than $1 \mathrm{meV} /$ molecule, $5 \times 10^{-3} \mathrm{GPa}$, and $10^{-3} \AA$, respectively. Using DFT-PBE we obtained a very accurate description of the free molecule (bond length and harmonic vibrational frequency within $0.5 \%$ and $1.5 \%$ of the experimental values, respectively) and of the Pa3 crystal structure. GGA calculations also correctly predict the polymerization of $\mathrm{CO}_{2}$ at high $P$.

[20] In the case of $P 4_{2} / \mathrm{mnm}$, the bond length is $1.154 \AA$ at $28 \mathrm{GPa}$, and eventually reduces to $1.148 \AA$ at $50 \mathrm{GPa}$.

[21] Our optimized Cmca structure differs slightly form the one reported experimentally [14] and agrees with previous theoretical works (F. Gygi, Comput. Mater. Sci. 10, 63 (1998), and Ref. 5).

[22] We find that the values of these parameters are sensitive to the choice of the functional form used to fit the energy as a function of volume, as observed for other solids. A single set of $P V$ data can be well fit with different combinations of $B_{0}$ and $B_{0}^{\prime}$ parameters; see, for example, N. R. Keskar et al., Phys. Rev. B 44, 4081 (1991). Nevertheless, their relative values are qualitatively the same irrespective of the fit.

[23] Phonon computations were carried out in the harmonic approximation using the linear response method: S. Baroni, P. Giannozzi, and A. Testa, Phys. Rev. Lett. 58, 1861 (1987); X. Gonze, Phys. Rev. B 55, 10337 (1997); ibid. 55, 10355 (1997); ibid. 56, 7321 (1997); ibid. 51, 8610 (1995). For phonon free energies, dynamical matrices were computed on a 64 point MP grid, corresponding 
to a real space cell containing 384 atoms (768 for $\mathrm{Pa} 3$ ).

[24] C.S. Yoo, private communications. 